\title{
Localization and Associative Strength of Acid Polysaccharides in Coccoliths of Pleurochrysis haptonemofera (Haptophyta) Predicted from Their Extractability from Partially Decalcified Coccoliths
}

\author{
Yasutaka Hirokawa $^{1,2}$, Satoru Matsuzuka ${ }^{1}$, Sho Itayama ${ }^{1}$, Tatsuya Uchida ${ }^{1}$, Shoko Fujiwara ${ }^{1}$, \\ Noriaki Ozaki $^{3,4}$, Hiromichi Nagasawa ${ }^{3}$, Mikio Tsuzuki ${ }^{1,5}$ \\ ${ }^{1}$ School of Life Sciences, Tokyo University of Pharmacy and Life Sciences, Hachioji, Japan \\ ${ }^{2}$ Faculty of Science and Engineering, Waseda University, Tokyo, Japan \\ ${ }^{3}$ Department of Applied Biological Chemistry, Graduate School of Agricultural and Life Sciences, \\ The University of Tokyo, Tokyo, Japan \\ ${ }^{4}$ Department of Biotechnology, Akita Prefectural University, Akita, Japan \\ ${ }^{3}$ Japan Science and Technology Agency, CREST, Tokyo, Japan \\ Email: fujiwara@toyaku.ac.jp
}

Received October 8, 2012; revised November 23, 2012; accepted December 11, 2012

\begin{abstract}
The coccolithophorids have calcified scales called coccoliths on their surface that include abundant acid polysaccharides. To determine the localization and associative strength of acid polysaccharides Ph-PS-1, -2 , and -3 in coccoliths of Pleurochrysis haptonemofera, we analyzed the acid polysaccharides extracted with urea from partially decalcified coccoliths. On treatment of coccoliths with $8.0 \mathrm{M}$ urea at $\mathrm{pH} 8.0$ in boiling water, Ph-PS-2 was not extracted, but parts of Ph-PS-1 and -3 were from the surface without the crystal morphology being affected. When coccoliths were partially decalcified at various $\mathrm{pHs}(\mathrm{pH} 8.0-5.0)$, Ph-PS-1, -3 , and -2 were extracted with urea in that order as the calcite crystals dissolved. Detection of the acid polysaccharides using fluorescein-isothiocyanate-labeled lectin (ConA) and anti-Ph-PS-2 antibodies demonstrated that Ph-PS-2 exists on the surfaces of both untreated and urea-treated coccoliths. Moreover, NaClO-treatment under moderate conditions extracted only Ph-PS-1, -3, and part of Ph-PS-2 from coccoliths, without separation of the crystal units, suggesting that at least some part of Ph-PS-2 acts as a glue connecting crystal units and/or a crystal unit and a base plate inside coccoliths. These results suggest that Ph-PS-2 exists not only on the surface but also inside of the coccoliths, most of it being strongly bound to coccoliths, while Ph-PS-1 and -3 are weakly associated close to the coccolith surface, in that order from the surface. This strongly supports a scheme in which PS-2 type acid polysaccharides play an important role in the crystal nucleation and PS-3 type functions during crystal growth.
\end{abstract}

Keywords: Acid Polysaccharide; Calcification; Coccolithophorid; Localization; Pleurochrysis haptonemofera; Urea-Extraction

\section{Introduction}

The coccolithophorids are unicellular microalgae belonging to the Haptophyta that have calcified scales, called coccoliths, on their surface. A coccolith in many coccolithophorides, including most extensively examined genera Pleurochrysis and Emiliania, consists of elaborate calcite crystals, a crystal coat comprising organic matter, and an organic base plate. The calcite crystals are composed of two distinct interlocking units, $\mathrm{V}$ and $\mathrm{R}$ units. Coccoliths are produced intracellularly in special organelles called coccolith vesicles. TEM observation of coccolith-forming cells has been extensively performed, and thereby the process of coccolith formation has been de- monstrated morphologically. However, it remains unclear how calcite crystals are formed and how the coccolith morphology is determined [1-3].

Biomineralization is a phenomenon that is observed in a wide variety of organisms, and in most cases, acidic organic matter such as acid proteins is involved in it [4]. In coccolithophorids, acid proteins have not been found in coccoliths, but acid polysaccharides are considered to participate in coccolith formation. Acid polysaccharides isolated previously showed high affinity to divalent cations $\mathrm{Ca}^{2+}$ (and $\mathrm{Mg}^{2+}$ ), and inhibitory activity toward $\mathrm{CaCO}_{3}$ precipitation in vitro $[5,6]$. In Pleurochrysis species, the existence of three types of acid polysaccharides 
has been reported [6-8]. From P. carterae and P. haptonemofera, acid polysaccharides, designated as PS-1, -2, and -3 , and Ph-PS-1, -2 (CMAP), and -3 , respectively, have been purified and characterized. The acid polysaccharides could not be solubilized from coccoliths by decalcification at low $\mathrm{pH}(\mathrm{pH} 5.5)$, while they were extractable with a divalent metal ion-chelating reagent, EDTA ( $\mathrm{pH} 8.0$ ), suggesting that they are bound to coccoliths via divalent metal ions, $\mathrm{Ca}^{2+}$ and/or $\mathrm{Mg}^{2+}$ [7]. Some chemically induced mutants and spontaneous variants of $P$. carterae, which are defective in coccolith formation, have been revealed to lack acid polysaccharide PS-2 or PS-3, suggesting that these acid polysaccharides play an important role in coccolith formation $[9,10]$. However, how they contribute to coccolith morphogenesis remains unclear. In this paper, we report results suggesting the localization and associative strength of acid polysaccharides in coccoliths of $P$. haptonemofera, and discuss how the acid polysaccharides are involved in coccolith formation.

\section{Materials and Methods}

\subsection{Algal Cells and Culture Conditions}

Coccolith-forming cells of $P$. haptonemofera were obtained and cultured axenically as described previously [11]. The cells were photoautotrophically grown in ESM medium [12] at $20^{\circ} \mathrm{C}$ under continuous illumination at 8 $\mathrm{W} \cdot \mathrm{m}^{-2}$ with constant bubbling of air.

\subsection{Isolation of Coccoliths}

The coccoliths liberated from the cell surface into the medium were isolated by the following procedure. A culture at the early-stationary phase was centrifuged, and then the white pellet of released coccoliths on the whitish brown pellet of cells was collected with the tip of a micropipet or a spatula and resuspended in $50 \mathrm{mM}$ HEPES buffer ( $\mathrm{pH}$ 8.0). Contaminating cells in the suspension were disrupted by sonication (at range 2 for $8 \mathrm{sec}$, using a Sonifier 250 D (Branson Ultrasonics Co., Danbury, CT, USA)), and then the suspension was passed through a Percoll (Amersham Pharmacia Biotech, Chalfont, UK) gradient $(1.2 \mathrm{ml}$ of $50 \%$ Percoll per $0.3 \mathrm{ml}$ of crude coccolith suspension). The purified coccolith pellet was washed with the HEPES buffer ( $\mathrm{pH} 8.0) . \mathrm{Ca}^{2+}$ included in the coccolith suspension was determined by the direct EDTA titration method, as described previously $[13,14]$.

\subsection{Denaturant- and pH-Treatments of Coccoliths}

For urea-treatment of coccoliths, isolated coccoliths containing $10 \mu \mathrm{mol} \mathrm{Ca}{ }^{2+}$ were suspended in $0.1 \mathrm{ml}$ of a urea solution (8.0 M urea, $100 \mathrm{mM}$ HEPES ( $\mathrm{pH} 8.0)$ ), and then boiled for $10 \mathrm{~min}$ in a water bath. For guanidine hydrochloride $(\mathrm{GdnHCl})$-treatment, 6.0 M GdnHCl was used in place of $8.0 \mathrm{M}$ urea. When various $\mathrm{pH}$-treatments were performed before the urea-treatment, the coccoliths (containing $10 \mu \mathrm{mol} \mathrm{Ca}{ }^{2+}$ ) were suspended in $0.5 \mathrm{ml}$ of $100 \mathrm{mM}$ HEPES or MES buffer (MES buffer, $\mathrm{pH} 5.0$ 6.5, or HEPES buffer, pH 7.0 - 8.0), and then mixed well by vigorous shaking for $30 \mathrm{~min}$. Sodium hypochlorite ( $\mathrm{NaClO}$ )-treatment was performed basically following the method of [15]. Isolated coccoliths (including $10 \mu \mathrm{mol}$ $\mathrm{Ca}^{2+}$ ) were suspended in $1.0 \mathrm{ml}$ of a $5 \%$ of $\mathrm{NaClO}$ solution, and then heated for $60 \mathrm{~min}$ at $85^{\circ} \mathrm{C}$. In some cases, $100 \mathrm{mM}$ HEPES-NaOH was added to the $\mathrm{NaClO}$ solution to lower its $\mathrm{pH}$ to 8.5 .

\subsection{Analysis of Acid Polysaccharides}

The extracted acid polysaccharides were quantified by the carbazole method [16] with glucuronic acid as a standard and then analyzed by PAGE. PAGE was performed on $20 \%(\mathrm{w} / \mathrm{v})$ gels [17], and acid polysaccharides were visualized with Stains-All (Hokudo) [18]. The unextracted acid polysaccharides were also analyzed after decalcification with $500 \mathrm{mM}$ EDTA (pH 8.0) and buffer replacement using a Vivaspin 500 (3,000 MWCO;- Sartorius AG, Göttingen, Germany) because EDTA hinders quantification and PAGE. Effective extraction needs 50 times as many EDTA molecules as $\mathrm{Ca}^{2+}$ ones included in the coccoliths as $\mathrm{CaCO}_{3}$.

Acid polysaccharides on the surface of the treated coccoliths were detected and quantified using fluoresceinisothiocyanate (FITC)-labeled lectin, ConA [7,14], and anti-CMAP (Ph-PS-2) antibodies. For detection with FITC-labeled ConA, $10 \mu \mathrm{g}$ of ConA was added to $200 \mu \mathrm{l}$ of a treated coccolith suspension, which had been prepared with coccoliths containing $10 \mu \mathrm{mol} \mathrm{Ca}^{2+}$, and then the mixture was incubated for $1 \mathrm{~h}$ in the dark. After centrifugation $\left(18,500 \mathrm{~g}, 5 \mathrm{~min}, 4^{\circ} \mathrm{C}\right)$, the pellet was washed twice with $200 \mu \mathrm{l}$ of $50 \mathrm{mM}$ Tris- $\mathrm{HCl}(\mathrm{pH} 8.0)$ to remove free lectin, and then dissolved in $300 \mu$ of $500 \mathrm{mM}$ EDTA ( $\mathrm{pH} 8.0$ ). After samples had been transferred to the wells of a 96-well microtiterplate, the relative fluorescence of each sample was measured with Fluoroskan II (Dainippon Pharmaceutical Co., Osaka, Japan).

For detection with anti-Ph-PS-2 antibodies, the ELISA method involving horseradish peroxidase (HRP)-linked secondary antibodies was performed. As the primary antibody, rabbit antiserum against Ph-PS-2, which was purified by application to a TSG-gel DEAE-5PW HPLC column (7.5 × $75 \mathrm{~mm}$; Tosoh, Tokyo, Japan) several times and whose purity was confirmed by NMR, was used [8]. One microliter of the antiserum was added to $200 \mu \mathrm{l}$ of a coccolith suspension (containing $4 \mu \mathrm{mol} \mathrm{Ca}{ }^{2+}$ of coccoliths) in the blocking buffer (non-fat dry milkcontaining $1 \times$ TBS $(0.05 \mathrm{M}$ Tris- $\mathrm{HCl}, 0.15 \mathrm{M} \mathrm{NaCl}, \mathrm{pH}$ 
8.0) centrifuged in advance to remove excess non-fat dry milk), and then the mixture was incubated for $1 \mathrm{~h}$ at room temperature. After centrifugation (2000 g, $5 \mathrm{~min}$ ), the pellet was washed with $1 \times \mathrm{TBS}$, and then the primary antibodies bound to the coccolith surface were detected using anti-rabbit IgG, HRP-linked secondary antibodies and TMB substrate (Cell Signaling Technology, Inc., Danvers, MA, USA), according to the suppliers.

\subsection{Scanning Electron Microscopy (SEM)}

The coccolith morphology was observed by SEM (JCM5700; JEOL, Tokyo, Japan), as described previously [19].

\section{Results and Discussion}

\subsection{Extraction of Acid Polysaccharides from Coccoliths with Urea}

On urea-treatment of isolated coccoliths, a part of the acid polysaccharides (parts of Ph-PS-1 and -3) was extracted (Figure 1, lane 2), whereas the proteins were not detectable in the extract even on silver-staining after SDS-PAGE. The sum of the acid polysaccharides extracted on urea-treatment and these subsequently extracted with EDTA was equal to that extracted with EDTA without the urea-treatment, suggesting that acid polysaccharides that are thoroughly extracted with EDTA are only partially extracted with urea (Figure 1, lanes 1-3). Similar partial extraction of acid polysaccharides was observed with a denaturant having an identical effect, GdnHCl (Figure 1, lanes 4 and 5). The urea- and GdnHCl-treatments neither dissolved $\mathrm{CaCO}_{3}$ nor affected the morphology of the coccoliths so much, as observed

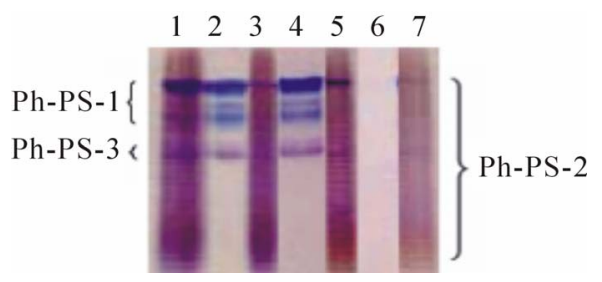

Figure 1. PAGE of acid polysaccharides extracted from coccoliths with various denaturing and oxidizing reagents. Acid polysaccharides were extracted from coccoliths with 8.0 M urea (lanes 2) and 6.0 M GdnHCl (lane 4) at pH 8.0 in boiling water, and then acid polysaccharides remaining in the coccoliths were completely extracted with EDTA (lanes 3 and 5, respectively). Acid polysaccharides remaining in the coccoliths after treatments with $5 \% \mathrm{NaClO}(\mathrm{pH} \geq 12.0$; lane 6) and buffered $\mathrm{NaClO}\left(\mathrm{pH} 8.5,5 \%\right.$; lane 7) at $85^{\circ} \mathrm{C}$ were completely extracted with EDTA. The extracts were analyzed by Stains-All staining after PAGE. Total acid polysaccharides extracted from non-treated coccoliths with EDTA were also analyzed as a control (lane 1). Acid polysaccharides extracted from a fixed amount of coccoliths were loaded in all lanes. Identical trends were observed in another experiment. on SEM (Figures 2(a)-(d), suggesting that the extracted acid polysaccharides were localized close to the coccolith surface. Thus, these treatments were expected to be novel methods applicable to analysis of the localization of acid polysaccharides in coccoliths.

\subsection{Urea-Extraction of Partially Decalcified Coccoliths}

For further investigation of localization of acid polysaccharides in coccoliths, the partially decalcified coccoliths were applied to urea-extraction. When exposed to low $\mathrm{pH}$ were subsequently treated with urea, the amount of extracted acid polysaccharides increased as the amount of dissolved $\mathrm{CaCO}_{3}$ increased (Figure 3). Ph-PS-1, -3,

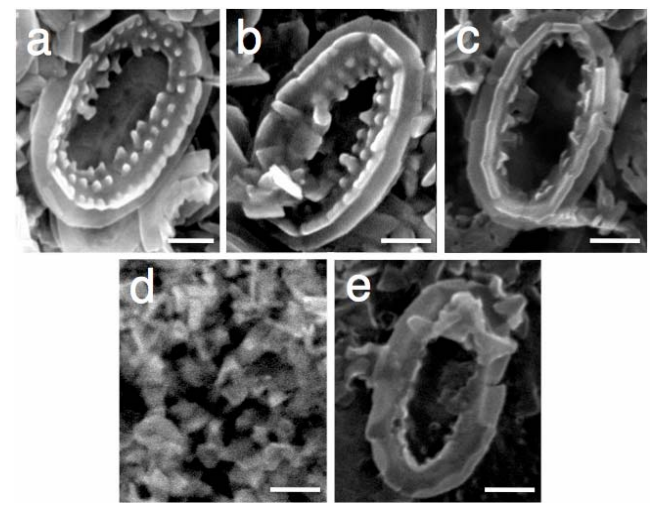

Figure 2. Scanning electron micrographs of coccoliths treated with urea. Coccoliths (a) were treated with urea (pH 8.0) (b), GdnHCl (pH 8.0) (c), NaClO (pH $\geq 12$ ) (d), and buffered $\mathrm{NaClO}$ (pH 8.5) (e), as described under Materials and Methods. Scale bars $=0.5 \mu \mathrm{m}$. Identical trends were observed in another experiment.

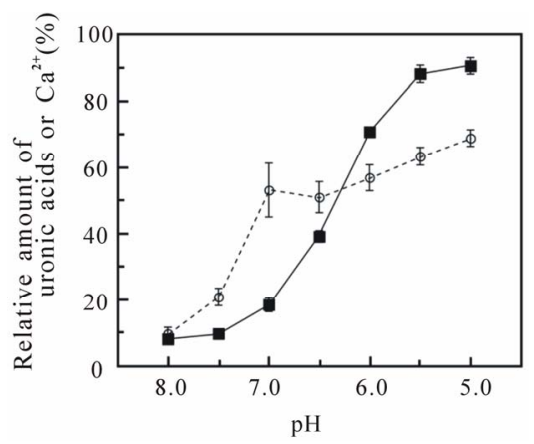

Figure 3. The relative amounts of uronic acids and $\mathrm{Ca}^{2+}$ solubilized from coccoliths on $\mathrm{pH}$ and urea treatments. Coccoliths that had been treated at $\mathrm{pH} 5.0$ - 8.0 were subsequently treated with $8.0 \mathrm{M}$ urea (pH 8.0), as described under Materials and Methods, and then the solubilized uronic acids $(\circ)$ and $\mathrm{Ca}^{2+}(\square)$ were quantified by the carbazole method [16] and the direct EDTA titration method $[13,14]$, respectively. Total uronic acids and $\mathrm{Ca}^{2+}$ solubilized with $500 \mathrm{mM}$ EDTA (pH 8.0) and $1 \mathrm{M} \mathrm{HCl}$, respectively, were also analyzed as controls. The data are the means \pm SD for three independent experiments. 
and -2 were extracted with urea, in that order, as the calcite crystals dissolved (Figure 4, lanes 4-8, and Figure 5), and Ph-PS-2 seemed to be extracted in order of molecular weight, from smaller to larger (Figure 4). This finding indicates that urea can attack more acid polysaccharides as $\mathrm{CaCO}_{3}$ dissolves, and suggests that parts of these acid polysacharides exist inside the coccoliths where the reagent cannot gain access when the calcite

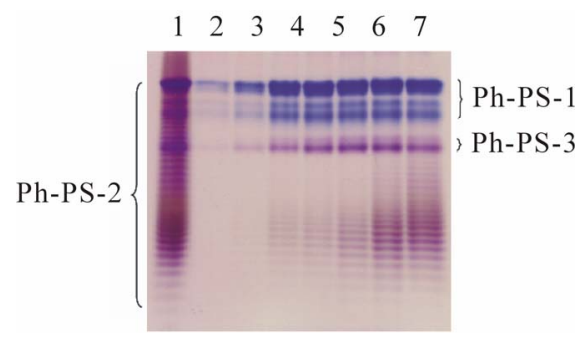

Figure 4. PAGE of acid polysaccharides extracted with urea from coccoliths that had been treated at various pHs. Acid polysaccharides were extracted by urea-treatment from

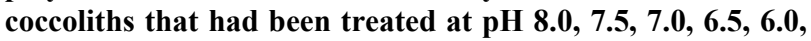
5.5, and 5.0 (lanes 2-8), and then the extracts were analyzed by Stains-all staining after PAGE, as described under Materials and Methods. Total acid polysaccharides extracted with $500 \mathrm{mM}$ EDTA (pH 8.0) were also analyzed as a control (lane 1). Acid polysaccharides extracted from a fixed amount of coccoliths were loaded in all lanes. Identical trends were observed in another experiment.

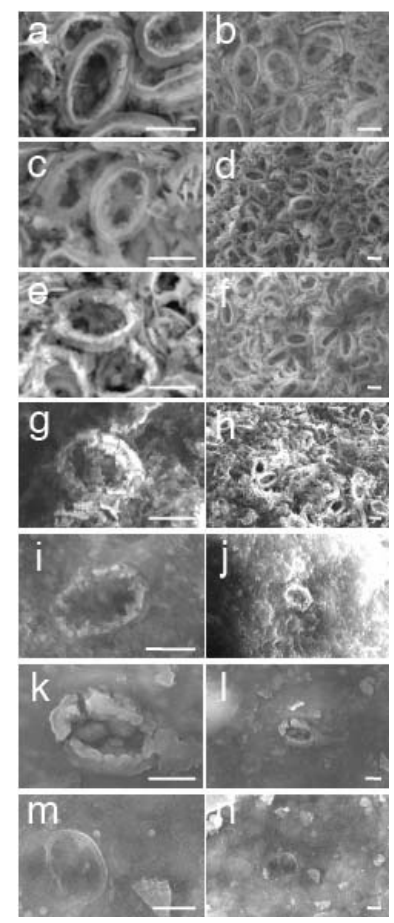

Figure 5. Scanning electron micrographs of coccoliths treated at pH 8.0 ((a) and (b)), 7.5 ((c) and (d)), 7.0 ((e) and (f)), $6.5((\mathrm{~g})$ and (h)), 6.0 ((i) and (j)), $5.5((\mathrm{k})$ and (l)), and 5.0 $((\mathrm{m})$ and $(\mathrm{n}))$ for $30 \mathrm{~min}$. Scale bars $=1 \mu \mathrm{m}$. Identical trends were observed in another experiment. crystals are intact.

When almost $40 \%$ of the $\mathrm{CaCO}_{3}$ in the coccoliths had been dissolved at $\mathrm{pH}$ 6.5, Ph-PS-1 and -3 were completely extracted with urea (Figures 3 and 4). The sharp increase in uronic acids in extracts from $\mathrm{pH} 8.0$ to 7.0 coincided with the extraction of Ph-PS-1 and -3 . On the other hand, more than half of the Ph-PS-2 remained in the coccoliths, even after they had been completely decalcified at $\mathrm{pH} 5.0$ and subsequently treated with urea (Figures 3 and 4). These findings suggest that Ph-PS-1 and -3 are weakly associated close to the coccolith surface, in that order from the surface, while some part of Ph-PS-2 (relatively small-size molecules) is weakly bound but exist deeply inside the coccoliths as if being protected from the detergent by $\mathrm{CaCO}_{3}$, the remainder of it (relatively large-size molecules) being strongly bound directly onto the base plate and/or in the crystal coat. The binding stability of Ph-PS-2 on coccoliths might be affected by the amount of associated divalent cations per molecule.

\subsection{NaClO-Treatment of Coccoliths}

To obtain a clue about the localization sites of Ph-PS-2, coccoliths were treated with the oxidizing reagent $\mathrm{NaClO}$, which separates crystals from each other [15], and it was confirmed that crystals were separated from each other (Figure 2(d)), and neither uronic acids nor acid polysaccharides remained in the crystals (Figure 1, lane 6). The addition of HEPES-buffer $(\mathrm{pH} 8.0$ ) to the $\mathrm{NaClO}$ solution, however, coincidentally repressed the degradation of acid polysaccharides (part of Ph-PS-2 remained in the treated coccoliths; Figure 1, lane 7) and the dissociation of crystal units (Figure 2(e)). These results suggested that part of Ph-PS-2 exists inside a coccolith, between crystal units and/or between crystal units and base plates as a molecular glue. Also, the fact that the oval shape of coccoliths was kept even after Ph-PS-1 and -3 had been completely removed from partially decalcified (at $\mathrm{pH}$ 6.5) coccoliths by urea-treatment (Figures 5(g), (h)) supports the importance of Ph-PS-2 for maintenance of the coccolith shape.

\subsection{Detection of Ph-PS-2 on the Coccolith Surface}

To determine whether or not Ph-PS-2 exists in the crystal coat and remains there even on urea-treatment, we tried to detect acid polysaccharides using FITC-labeled ConA [7] and anti-Ph-PS-2 antibodies. First, the existence of acid polysaccharides on the coccolith surface was examined using FITC-labeled ConA, which binds to the cell surface, total acid polysaccharides extracted from there with EDTA, and purified Ph-PS-2, but not to the remnant's cell surface [7]. ConA bound to the coccolith sur- 
face, and the amount of ConA bound to the coccolith surface did not decrease so much $(72 \% \pm 17 \%$ (mean \pm $\mathrm{SD}, n=4)$ ) on urea-treatment (the small decrease was supposed to be due to partial extraction of Ph-PS-1 and/ or -3 , because the specificity of ConA was not so high for Ph-PS-2 (Figure 4, lane 2) [7]). This finding suggests that at least some acid polysaccharides are localized on the crystal coat surface and that a large part of them remains there even on urea-treatment.

Then, to more determine whether or not Ph-PS-2 exists on the coccolith surface, anti-Ph-PS-2 antibodies were used. Its binding to the coccolith surface could be detected by the modified ELISA method sensitively and the amount was not decreased by urea-treatment $(122 \% \pm$ 9\%; mean $\pm \mathrm{SD}, n=6$ ) (Table 1), suggesting that $\mathrm{Ph}-\mathrm{PS}-2$ exists on the coccolith surface and remains there even on such treatment. Similarly, in a closely related species, P. carterae, immunoelectron microscopy involving anti-PS-2 antibodies has revealed the existence of PS-2 in the crystal coat [15].

\subsection{Discussion on Localization and Functions of Acid Polysaccharides}

Marsh et al. [10,15] have found that PS-1 and PS-2 are localized in the crystal coats of mature coccoliths and in electron-dense Golgi particles (often called coccolithosomes), while PS-3 is localized at the interface between growing crystals and the membranes of coccolith vesicles, using anti-polysaccharide antibodies. Based on these findings, and the putative functions of PS-2 and PS-3 predicted on the observation of the immature coccolith morphology in variants devoid of acid polysaccharides $[9,10]$, they proposed the following interesting scheme for the coccolith formation process in P. carterae [2]: PS-1 and PS-2 form $20 \mathrm{~nm}$ coccolithosomes with $\mathrm{Ca}^{2+}$ and become attached to the base plate rim. PS-2 apparently acts as a $\mathrm{Ca}^{2+}$ buffer and facilitates calcite crystal nucleation there. The particles on the rim are displaced by a ring of small calcite crystals. PS-3 is required for the growth process. At the end of the calcite growth phase,

Table 1. Amount of Ph-PS-2 on the coccolith surface detected with the modified ELISA method involving anti-PhPS-2 antibodies ${ }^{\mathrm{a}}$.

\begin{tabular}{ccc}
\hline \multirow{2}{*}{ Coccoliths } & \multicolumn{2}{c}{ HRP activity (\%) } \\
\cline { 2 - 3 } & Preimmune serum & $\begin{array}{c}\text { Anti-Ph-PS-2 } \\
\text { antiserum }\end{array}$ \\
\hline Control (untreated) & $4 \pm 0$ & $100 \pm 1$ \\
Urea-treated & $7 \pm 1$ & $122 \pm 9$ \\
\hline
\end{tabular}

${ }^{\mathrm{a}} \mathrm{Ph}-\mathrm{PS}-2$ on the surface of urea-treated and untreated coccoliths was detected with anti-Ph-PS-2 antibodies. A one-hundredth to one-tenth volume of each antigen-antibody reaction mixture containing $4 \mu \mathrm{mol} \mathrm{Ca}{ }^{2+}$ of coccoliths was used for the detection with the HRP-linked secondary antibodies. Data are means $\pm \mathrm{SD}(n=3-6)$. the crystals acquire an amorphous polysaccharide coat derived through the dissociation of PS-1/PS-2-containing coccolithosomes.

On the other hand, in this study, the localization of acid polysaccharides in coccoliths was examined by a new extraction method involving denaturants that do not appear to decalcify them. Our results suggested that $\mathrm{Ph}-$ PS-1 and -3 are localized close to the coccolith surface, while at least some Ph-PS-2 is buried deep in the coccoliths. This finding supports her idea that Ph-PS-2 plays an important role in the crystal nucleation and Ph-PS-3 functions during the growth phase. Also, our results suggest that most Ph-PS-2 is strongly associated with the coccoliths, probably with both the coccolith surface and the base plate, and that a little of it is incorporated between crystal units and/or between crystal units and base plates (Figures 4 and 5). Furthermore, Ph-PS-3 is suggested to be incorporated at a relatively late stage of crystal growth, i.e., a little before the attachment of PS-1 (Figure 1, lanes 3 and 4). These ideas are well consistent with Marsh's scheme.

However, whether or not Ph-PS-1 and Ph-PS-2, both being included in coccolithosomes [15], are colocalized remains unclear. Since PS-1 and PS-2 are both included in coccolithosomes, they are expected to be colocalized inside coccoliths (directly on the base plate and between crystals), as well as on the surface. In this study, a result that seemed to suggest that Ph-PS-1 is localized more on the surface was obtained (Figure 4). However, this may be just due to a difference in extractability on urea-treatment between the two acid polysaccharides (Figure 1), although the possibility that Ph-PS-1 dissociates from the rim of the base plate before crystal nucleation or that the coccolithosome composition changes during coccolith maturation cannot be ruled out. The molecular mechanism, including the expression of the genes that regulate the biosynthesis and metabolism of acid polysaccharides, should be explored to clarify the mineralization process.

\section{Conclusion}

In this paper, the localization and associative strength of acid polysaccharides in coccoliths were investigated, using novel extraction method, urea-extraction, which could extract part of acid polysaccharides without coccolith decalcification. The results obtained by urea-extraction and other denaturant treatment indicated that the three acid polysaccharides are not localized uniformly: Ph-PS- 1 and -3 are weakly associated close to the coccolith surface, while Ph-PS-2 exists not only on the surface but also inside of the coccoliths, most of it being strongly bound to coccoliths. The results also suggested that $\mathrm{Ph}-$ PS-2 plays an important role in maintenance of coccolith oval shape. These strongly support a scheme in which PS-2 type acid polysaccharides play an important role in 
the crystal nucleation and PS-3 type functions during crystal growth.

\section{Acknowledgements}

We are grateful to Drs. I. Inouye and M. Kawachi of Tsukuba University for kindly providing the $P$. haptonemofera cells, to Mr. H. Akiyama of Tokyo University of Pharmacy and Life Sciences for the kind support in the SEM observations and to Mr. N. J. Halewood for correcting the English version of this paper. This work was supported by a Grant-in-Aid from the Ministry of Education, Science, Sports and Culture, Japan (grant nos. 20570059 and 24570114).

\section{REFERENCES}

[1] E. Paasche, "A Review of the Coccolithophorid Emiliania huxleyi (Prymnesiophyceae), with Particular Reference to Growth, Coccolith Formation, and Calcification-Photosynthesis Interactions," Phycologia, Vol. 40, No. 6, 2001, pp. 503-529. doi: 10.2216/i0031-8884-40-6-503.1

[2] M. E. Marsh, "Regulation of $\mathrm{CaCO}_{3}$ Formation in Coccolithophores," Comparative Biochemistry and Physiology, Part B: Biochemistry and Molecular Biology, Vol. 136, No. 4, 2003, pp. 743-754. doi:10.1016/S1096-4959(03)00180-5

[3] Y. Shiraiwa, "Physiological Regulation of Carbon Fixation in the Photosynthesis and Calcification of Coccolithophorids," Comparative Biochemistry and Physiology, Part B: Biochemistry and Molecular Biology, Vol. 136, No. 4, 2003, pp. 775-783. doi:10.1016/S1096-4959(03)00221-5

[4] H. Nagasawa, "Macromolecules in Biominerals of Aquatic Organisms," Thalassas, Vol. 20, No. 1, 2004, pp. 1524.

[5] A. H. Borman, E. W. de Jong, M. Huizinga, D. J. Kok, P. Westbroek and L. Bosch, "The Role in $\mathrm{CaCO}_{3}$ Crystallization of an Acid $\mathrm{Ca}^{2+}$-Binding Polysaccharide Associated with Coccoliths of Emiliania huxleyi," European Journal of Biochemistry, Vol. 129, No. 1, 1982, pp. 179-183. doi:10.1111/j.1432-1033.1982.tb07037.x

[6] M. E. Marsh, D. K. Chang and G. C. King, "Isolation and Characterization of a Novel Acidic Polysaccharide Containing Tartrate and Glyoxylate Residues from the Mineralized Scales of a Unicellular Coccolithophorid Alga Pleurochrysis carterae," Journal of Biological Chemistry, Vol. 267, No. 28, 1992, Article ID: 20507-20512.

[7] Y. Hirokawa, S. Fujiwara and M. Tsuzuki, "Three Types of Acidic Polysaccharides Associated with Coccolith of Pleurochrysis haptonemofera: Comparison of the Biochemical Characteristics with Those of $P$. carterae and Analysis Using Fluorescein-Isothiocyanate-Labeled Lectins," Marine Biotechnology, Vol. 7, No. 6, 2005, pp. 634-644. doi:10.1007/s10126-004-5148-9

[8] N. Ozaki, S. Sakuda and H. Nagasawa, "A Novel Highly Acidic Polysaccharide with Inhibitory Activity on Calcification from the Calcified Scale 'Coccolith' of a Coccolithophorid Alga, Pleurochrysis haptonemofera," Bioche- mical and Biophysical Research Communications, Vol. 357, No. 4, 2007, pp. 1172-1176. doi:10.1016/j.bbrc.2007.04.078

[9] M. E. Marsh and D. P. Dickinson, "Polyanion-Mediated Mineralization-Mineralization in Coccolithophore (Pleurochrysis carterae) Variants Which Do Not Express PS2, the Most Abundant and Acidic Mineral-Associated Polyanion in Wild-Type Cells," Protoplasma, Vol. 199, No. 1-2, 1997, pp. 9-17. doi:10.1007/BF02539801

[10] M. E. Marsh, A. L. Ridall, P. Azadi and P. J. Duke, "Galacturonomannan and Golgi-derived Membrane Linked to Growth and Shaping of Biogenic Calcite," Journal of Structural Biology, Vol. 139, No. 1, 2002, pp. 39-45. doi:10.1016/S1047-8477(02)00503-8

[11] S. Fujiwara, Y. Hirokawa, Y. Takatsuka, K. Suda, E. Asamizu, T. Takayanagi, D. Shibata, S. Tabata and M. Tsuzuki, "Gene Expression Profiling of Coccolith-Bearing Cells and Naked Cells in Haptophyte Pleurochrysis haptonemofera with a cDNA Macroarray System," Marine Biotechnology, Vol. 9, No. 5, 2007, pp. 550-560. doi:10.1007/s10126-007-9039-8

[12] M. M. Watanabe, F. Kasai and R. Sudo, "NIES-Collection List of Strains, 2nd Edition, Microalgae and Protozoa," The National Institute for Environmental Studies, Tsukuba, 1988.

[13] J. Patton and W. Reeder, "New Indicator for Titration of Calcium with (Ethylenedinitrilo) Tetraacetate," Analytical Chemistry, Vol. 28, No. 6, 1956, pp. 1026-1028. doi:10.1021/ac60114a029

[14] J. Takahashi, S. Fujiwara, M. Kikyo, Y. Hirokawa and M. Tsuzuki, "Discrimination of the Cell Surface of the Coccolithophorid Pleurochrysis haptonemofera from Light Scattering and Fluorescein-Isothiocyanate-Labeled Lectin Staining Measured by Flow Cytometry," Marine Biotechnology, Vol. 4, No. 1, 2002, pp. 94-101. doi:10.1007/s10126-001-0083-5

[15] M. E. Marsh, "Polyanion-Mediated Mineralization- Assembly and Reorganization of Acidic Polysaccharides in the Golgi System of a Coccolithophorid Alga during Mineral Deposition," Protoplasma, Vol. 177, No. 3-4, 1994, pp. 108-122. doi:10.1007/BF01378985

[16] T. Bitter and H. M. Muir, "A Modified Uronic Acid Carbazole Reaction," Analytical Biochemistry, Vol. 4, No. 4, 1962, pp. 330-334. doi:10.1016/0003-2697(62)90095-7

[17] U. K. Laemmli, "Cleavage of Structural Proteins during the Assembly of the Head of Bacteriophage T4," Nature, Vol. 227, No. 5259, 1970, pp. 680-685. doi: $10.1038 / 227680 \mathrm{a} 0$

[18] M. R. Green, J. V. Pastewka and A. Peacock, "Differential Staining of Phosphoproteins on Polyacrylamide Gels with a Cationic Carbocyanine Dye," Analytical Biochemistry, Vol. 56, No. 1, 1973, pp. 43-51. doi:10.1016/0003-2697(73)90167-X

[19] F. Katagiri, Y. Takatsuka, S. Fujiwara and M. Tsuzuki, "Effects of $\mathrm{Ca}$ and $\mathrm{Mg}$ on Growth and Calcification of the Coccolithophorid Pleurochrysis haptonemofera: Ca Requirement for Cell Division in Coccolith-Bearing Cells and for Normal Coccolith Formation with Acidic Polysaccharides," Marine Biotechnology, Vol. 12, No. 1, 2010 , pp. $42-51$. 